Article

\title{
Genetic Diversity among Some Walnut (Juglans regia L.) Genotypes by SSR Markers
}

\author{
Murat Guney ${ }^{1, *(\mathbb{D}}$, Salih Kafkas ${ }^{2}$, Hakan Keles ${ }^{1}$, Mozhgan Zarifikhosroshahi ${ }^{2}$, Muhammet Ali Gundesli ${ }^{3}$, \\ Sezai Ercisli ${ }^{4}\left(\mathbb{D}\right.$, Tomas Necas ${ }^{5}\left({ }^{\circ}\right.$ and Geza Bujdoso ${ }^{6}(\mathbb{C}$
}

1 Department of Horticulture, Faculty of Agriculture, Yozgat Bozok University, Yozgat 66900, Turkey; hakan.keles@yobu.edu.tr

2 Department of Horticulture, Faculty of Agriculture, Cukurova University, Adana 01380, Turkey; salihkafkas@gmail.com (S.K.); mn_zarifi@yahoo.com (M.Z.)

3 Department of Horticulture, East Mediterranean Transitional Zone Agricultural Research Institute, Kahramanmaras 46140, Turkey; muhammetali.gundesli@tarimorman.gov.tr

4 Department of Horticulture, Faculty of Agriculture, Ataturk University, Erzurum 25240, Turkey; sercisli@atauni.edu.tr

5 Department of Fruit Science, Faculty of Horticulture in Lednice, Mendel University in Brno, Valticka 337, 69144 Lednice, Czech Republic; tomas.necas@mendelu.cz

6 Institute of Horticultural Science, Hungarian University of Agriculture and Life Science, Villányi Str. 29-43, 1118 Budapest, Hungary; resinfru@yahoo.com

* Correspondence: murat.guney@yobu.edu.tr; Tel.: +90-354-242-10-28

check for updates

Citation: Guney, M.; Kafkas, S.; Keles, H.; Zarifikhosroshahi, M.; Gundesli, M.A.; Ercisli, S.; Necas, T.; Bujdoso, G. Genetic Diversity among Some Walnut (Juglans regia L.) Genotypes by SSR Markers. Sustainability 2021, 13, 6830. https:// doi.org/10.3390/su13126830

Academic Editors: Jun-Ichi Sakagami and Marco Lauteri

Received: 27 April 2021

Accepted: 4 June 2021

Published: 17 June 2021

Publisher's Note: MDPI stays neutral with regard to jurisdictional claims in published maps and institutional affiliations.

Copyright: (c) 2021 by the authors. Licensee MDPI, Basel, Switzerland. This article is an open access article distributed under the terms and conditions of the Creative Commons Attribution (CC BY) license (https:// creativecommons.org/licenses/by/ $4.0 /)$.

\begin{abstract}
The food needs for increasing population, climatic changes, urbanization and industrialization, along with the destruction of forests, are the main challenges of modern life. Therefore, it is very important to evaluate plant genetic resources in order to cope with these problems. Therefore, in this study, a set of ninety-one walnut (Juglans regia L.) accessions from Central Anatolia region, composed of seventy-four accessions and eight commercial cultivars from Turkey, and nine international reference cultivars, was analyzed using 45 SSR (Simple Sequence Repeats) markers to reveal the genetic diversity. SSR analysis identified 390 alleles for 91 accessions. The number of alleles per locus ranged from 3 to 19 alleles with a mean value of 9 alleles per locus. Genetic dissimilarity coefficients ranged from 0.03 to 0.68 . The highest number of alleles was obtained from CUJRA212 locus $(\mathrm{Na}=19)$. The values of polymorphism information content $(\mathrm{PIC})$ ranged from 0.42 (JRHR222528) to 0.86 (CUJRA212) with a mean PIC value of 0.68. Genetic distances were estimated according to the UPGMA (Unweighted Pair Group Method with Arithmetic Average), Principal Coordinates (PCoA), and the Structure-based clustering. The UPGMA and Structure clustering of the accessions depicted five major clusters supporting the PCoA results. The dendrogram revealed the similarities and dissimilarities among the accessions by identifying five major clusters. Based on this study, SSR analyses indicate that Yozgat province has an important genetic diversity pool and rich genetic variance of walnuts.
\end{abstract}

Keywords: Juglans regia; genetic diversity; SSR; PCoA; UPGMA; structure analysis

\section{Introduction}

The walnut plant, botanically known as Juglans regia L., is categorized in genus Juglans and belongs to the Juglandaceae family. The Juglans genus has more than 20 species [1]. J. regia L., known as the common walnut (English or Persian walnut), is a long-lived, deciduous, monoecious, open-pollinated, and generally dichogamous plant. J. regia is a diploid plant with a haploid chromosome number of $n=16$ [2].

Numerous studies have been conducted on walnut fruits concerning biochemical, phytochemical, and antioxidant characteristics, and their contribution to human health and nutrition. Walnut kernel has a high amount of protein, fat, vitamins, minerals, and polyphenols, therefore, placing walnut in the human diet is inevitable [3,4]. Walnut has 
been also using in folk medicine due to its high antioxidant capacity [5] and alkaloids content with antimicrobial properties and $\omega-3$ fatty acid concentration [6] for a long time. Total world production of walnut has reached about 3,829,626 tons in 2017 [7], and China, the USA, Iran, Turkey, Mexico, and Ukraine are the main walnut producer countries in the world. To raise walnut production and exportation, new genotypes should be introduced, and old genotypes should be also preserved in gene banks [8]. For achieving this goal, the foremost step is the detection of superior genotypes and then the characterization of their genetic variability. In addition, walnut is a precious industrial plant, especially the ones grown wildly in the forest. However, the conservation of forest genetic resources and the improvement of our forest trees are also of vital importance for sustainable forestry [9]. On the other hand, the breeding work in wild walnut trees is a slow process due to the large genome structures and the length of the reproduction period of the trees. However, the rapid progress in today's technologies helps to shorten the breeding period [10].

Molecular markers are based on DNA variation, which in plant cells is the nucleus (nuclear genome) and in organelles like plastids and mitochondria. Molecular markers are evaluated with high reliability in determining diversity among plant population and also plant genotypes in the population. Briefly, molecular markers facilitate to establish the relationship between phenotypic and genotypic diversity [11]. Today, molecular markers have been efficient tools for the description of genetic differences among plants. Therefore, molecular markers are suggested to explore genetic diversity in walnut. To date, many molecular marker technologies have been developed and have been used in studies such as identification, characterization, genetic mapping, marker-based selection, etc. of plant cultivars and accessions [10-14]. Among PCR-based molecular markers used to determine genetic relationships in plants, SSRs are preferred due to their advantages of being codominant, redundancy in the genome, being high polymorphism, and repeatability. SSR markers have been applied for molecular characterization of different species such as hawthorn [11], quince [13], terebinth [14], apricot [15], apple [16], pear [17], and pistachio [18]. The first SSR marker development study in walnut was done by Woeste et al. [19], following studies are Victory et al. [20], Hoban et al. [21], Zhang et al. [22], Feng et al. [23], Zhang et al. [24], Najafi et al. [25], Topcu et al. [26,27], Ikhsan et al. [28], Dang et al. [29], and Eser et al. [30]. Based on these studies, SSR markers have been extremely reliable in the determination of the relationship among walnut accessions. Moreover, SSR markers are also used in the investigation of the evolutionary relationship of species and populations, and in the examination of taxonomic relationships [31].

Walnuts are important genetic materials in conservation of genetic resources and in breeding studies. It is important to determine the genetic pedigree of selected walnut accessions and cultivars, to make the selection of new generations effectively, and to reveal the genetic background of walnut populations [10]. Although Central Anatolia contains valuable genetic resources, the genetic characterization of wild walnuts growing in this region has been neglected. Therefore, in the present study, it was focused on genetic diversity and relationships among some commercial walnut cultivars and accessions from different selected districts of Yozgat province using SSR markers.

\section{Materials and Methods}

\subsection{Plant Material}

A set of ninety-one accessions were used as experimental materials. Seventy-four accessions were from different geographical zones of Yozgat provinces located in Central Anatolia with old forests (Figure 1; Table 1), and 8 commercial cultivars from Turkey and 9 international reference cultivars cultivated in the "Nuts Application and Research Center (SEKAMER)", Kahramanmaras, Turkey. 


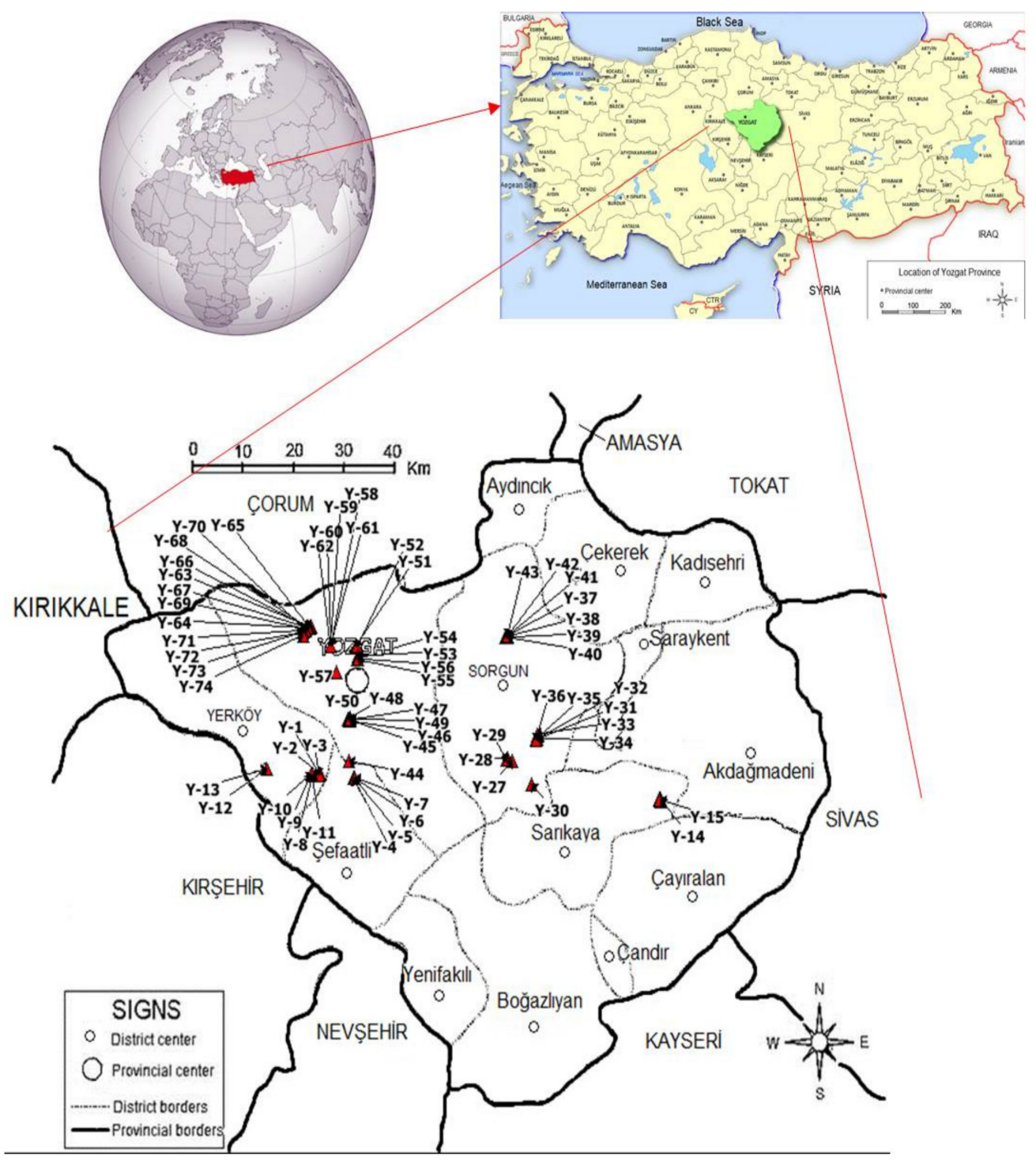

Figure 1. Map of the sampled areas highlighted by different colors and dots.

Table 1. The studied accessions of Juglans regia L. and their origins, latitude, longitude, and altitude.

\begin{tabular}{|c|c|c|c|c|c|c|c|c|}
\hline No & Location & $\begin{array}{c}\text { Sample } \\
\text { Code }\end{array}$ & Accessions & $\begin{array}{c}\text { Country of } \\
\text { Origin }\end{array}$ & Latitude & Longitude & $\begin{array}{l}\text { Altitude } \\
\text { (m) }\end{array}$ & $\begin{array}{c}\text { Tree Age } \\
\text { (year) }\end{array}$ \\
\hline 1 & \multirow{17}{*}{$\begin{array}{l}\text { SEKAMER } \\
\text { Turkey }\end{array}$} & ${ }^{*}$ Chandler & *Chandler & USA & - & - & - & - \\
\hline 2 & & *Fernor & ${ }^{*}$ Fernor & French & - & - & - & - \\
\hline 3 & & *Franquette & ${ }^{*}$ Franquette & French & - & - & - & - \\
\hline 4 & & ${ }^{*}$ Fernette & ${ }^{*}$ Fernette & French & - & - & - & - \\
\hline 5 & & ${ }^{*}$ Midland & *Midland & USA & - & - & - & - \\
\hline 6 & & *Pedro & *Pedro & USA & - & - & - & - \\
\hline 7 & & *Serr-1 & *Serr-1 & USA & - & - & - & - \\
\hline 8 & & *Amigo & *Amigo & USA & - & - & - & - \\
\hline 9 & & *Back & *Back & Unknown & - & - & - & - \\
\hline 10 & & *Kaplan-86 & *Kaplan-86 & Turkey & - & - & - & - \\
\hline 11 & & *Maraş-18 & ${ }^{*}$ Maraş-18 & Turkey & - & - & - & - \\
\hline 12 & & *Sütyemez-1 & *Sütyemez-1 & Turkey & - & - & - & - \\
\hline 13 & & *Şebin-1 & *Şebin-1 & Turkey & - & - & - & - \\
\hline 14 & & *Bilecik-2 & *Bilecik-2 & Turkey & - & - & - & - \\
\hline 15 & & *Yalova-1 & *Yalova-1 & Turkey & - & - & - & - \\
\hline 16 & & *Bursa-95 & *Bursa-95 & Turkey & - & - & - & - \\
\hline 17 & & *Kaman-1 & *Kaman-1 & Turkey & - & - & - & - \\
\hline
\end{tabular}


Table 1. Cont.

\begin{tabular}{|c|c|c|c|c|c|c|c|c|}
\hline No & Location & $\begin{array}{l}\text { Sample } \\
\text { Code }\end{array}$ & Accessions & $\begin{array}{c}\text { Country of } \\
\text { Origin }\end{array}$ & Latitude & Longitude & $\begin{array}{l}\text { Altitude } \\
\text { (m) }\end{array}$ & $\begin{array}{c}\text { Tree Age } \\
\text { (year) }\end{array}$ \\
\hline 18 & & Yozgat-1 & Ekinciusagi-1 & Turkey & $39^{\circ} 35^{\prime} 40.34^{\prime \prime}$ & $34^{\circ} 42^{\prime} 24.56^{\prime \prime}$ & 1155 & 80-100 \\
\hline 19 & & Yozgat-2 & Ekinciusagi-2 & Turkey & $39^{\circ} 35^{\prime} 45.25^{\prime \prime}$ & $34^{\circ} 42^{\prime} 21.20^{\prime \prime}$ & 1157 & $100-120$ \\
\hline 20 & Sefaatli & Yozgat-3 & Ekinciusagi-3 & Turkey & $39^{\circ} 35^{\prime} 36.69^{\prime \prime}$ & $34^{\circ} 42^{\prime} 20.78^{\prime \prime}$ & 1153 & 100-120 \\
\hline 27 & Yozgat & Yozgat-10 & Seydiyar-10 & Turkey & $39^{\circ} 35^{\prime} 27.74^{\prime \prime}$ & $34^{\circ} 47^{\prime} 58.16^{\prime \prime}$ & 1091 & $50-60$ \\
\hline 28 & Turkey & Yozgat-11 & Seydiyar-11 & Turkey & $39^{\circ} 35^{\prime} 27.84^{\prime \prime}$ & $34^{\circ} 47^{\prime} 58.24^{\prime \prime}$ & 1091 & $50-60$ \\
\hline 29 & & Yozgat-12 & Seydiyar-12 & Turkey & $39^{\circ} 35^{\prime} 22.96^{\prime \prime}$ & $34^{\circ} 48^{\prime} 5.45^{\prime \prime}$ & 1097 & $50-60$ \\
\hline 30 & & Yozgat-13 & Seydiyar-13 & Turkey & $39^{\circ} 35^{\prime} 21.43^{\prime \prime}$ & $34^{\circ} 48^{\prime} 6.08^{\prime \prime}$ & 1114 & $50-60$ \\
\hline 21 & & Yozgat-4 & Koycu-4 & Turkey & $39^{\circ} 35^{\prime} 30.11^{\prime \prime}$ & $34^{\circ} 41^{\prime} 14.01^{\prime \prime}$ & 1095 & $150-200$ \\
\hline 22 & & Yozgat-5 & Koycu-5 & Turkey & $39^{\circ} 35^{\prime} 34.66^{\prime \prime}$ & $34^{\circ} 40^{\prime} 59.95^{\prime \prime}$ & 1083 & 80-100 \\
\hline 23 & Yerkoy & Yozgat-6 & Koycu-6 & Turkey & $39^{\circ} 35^{\prime} 35.19^{\prime \prime}$ & $34^{\circ} 40^{\prime} 57.15^{\prime \prime}$ & 1077 & 150-200 \\
\hline 24 & Yozgat & Yozgat-7 & Koycu-7 & Turkey & $39^{\circ} 35^{\prime} 34.92^{\prime \prime}$ & $34^{\circ} 40^{\prime} 55.63^{\prime \prime}$ & 1072 & $100-120$ \\
\hline 25 & & Yozgat-8 & Kahyakoyu-8 & Turkey & $39^{\circ} 36^{\prime} 3.25^{\prime \prime}$ & $34^{\circ} 33^{\prime} 39.93^{\prime \prime}$ & 802 & $40-50$ \\
\hline 26 & & Yozgat-9 & Kahyakoyu-9 & Turkey & $39^{\circ} 36^{\prime} 4.29^{\prime \prime}$ & $34^{\circ} 33^{\prime} 37.48^{\prime \prime}$ & 804 & $40-50$ \\
\hline 42 & & Yozgat-26 & Hisarbey-26 & Turkey & $39^{\circ} 34^{\prime} 4.65^{\prime \prime}$ & $35^{\circ} 38^{\prime} 39.85^{\prime \prime}$ & 1247 & 150-200 \\
\hline 43 & & Yozgat-27 & Hisarbey-27 & Turkey & $39^{\circ} 34^{\prime} 4.77^{\prime \prime}$ & $35^{\circ} 38^{\prime} 40.19^{\prime \prime}$ & 1247 & 150-200 \\
\hline 44 & & Yozgat-28 & Hisarbey-28 & Turkey & $39^{\circ} 34^{\prime} 4.36^{\prime \prime}$ & $35^{\circ} 38^{\prime} 39.67^{\prime \prime}$ & 1247 & 150-200 \\
\hline 45 & & Yozgat-29 & Hisarbey-29 & Turkey & $39^{\circ} 34^{\prime} 4.22^{\prime \prime}$ & $35^{\circ} 38^{\prime} 40.05^{\prime \prime}$ & 1247 & 150-200 \\
\hline 46 & & Yozgat-30 & Hisarbey-30 & Turkey & $39^{\circ} 34^{\prime} 3.68^{\prime \prime}$ & $35^{\circ} 38^{\prime} 44.09^{\prime \prime}$ & 1252 & 150-200 \\
\hline 47 & Sarikaya & Yozgat-31 & Hisarbey-31 & Turkey & $39^{\circ} 34^{\prime} 4.48^{\prime \prime}$ & $35^{\circ} 38^{\prime} 41.44^{\prime \prime}$ & 1249 & 150-200 \\
\hline 48 & Yozgat & Yozgat-32 & Hisarbey-32 & Turkey & $39^{\circ} 33^{\prime} 58.49^{\prime \prime}$ & $35^{\circ} 38^{\prime} 44.14^{\prime \prime}$ & 1256 & 150-200 \\
\hline 49 & Turkey & Yozgat-33 & Hisarbey-33 & Turkey & $39^{\circ} 34^{\prime} 13.17^{\prime \prime}$ & $35^{\circ} 38^{\prime} 47.30^{\prime \prime}$ & 1257 & 150-200 \\
\hline 50 & & Yozgat-34 & Hisarbey-34 & Turkey & $39^{\circ} 34^{\prime} 15.82^{\prime \prime}$ & $35^{\circ} 38^{\prime} 45.05^{\prime \prime}$ & 1254 & $150-200$ \\
\hline 51 & & Yozgat-35 & Hisarbey-35 & Turkey & $39^{\circ} 34^{\prime} 15.47^{\prime \prime}$ & $35^{\circ} 38^{\prime} 38.96^{\prime \prime}$ & 1250 & 150-200 \\
\hline 52 & & Yozgat-36 & Hisarbey-36 & Turkey & $39^{\circ} 34^{\prime} 15.04^{\prime \prime}$ & $35^{\circ} 38^{\prime} 35.01^{\prime \prime}$ & 1248 & 150-200 \\
\hline 53 & & Yozgat-37 & Hisarbey-37 & Turkey & $39^{\circ} 34^{\prime} 14.75^{\prime \prime}$ & $35^{\circ} 38^{\prime} 32.07^{\prime \prime}$ & 1248 & 150-200 \\
\hline 54 & & Yozgat-38 & Hisarbey-38 & Turkey & $39^{\circ} 34^{\prime} 11.14^{\prime \prime}$ & $35^{\circ} 38^{\prime} 32.64^{\prime \prime}$ & 1243 & $150-200$ \\
\hline 38 & & Yozgat-21 & Karaveli-21 & Turkey & $39^{\circ} 38^{\prime} 5.64^{\prime \prime}$ & $35^{\circ} 14^{\prime} 4.31^{\prime \prime}$ & 1107 & $80-100$ \\
\hline 39 & & Yozgat-23 & Karaveli-23 & Turkey & $39^{\circ} 38^{\prime} 22.36^{\prime \prime}$ & $35^{\circ} 13^{\prime} 24.83^{\prime \prime}$ & 1081 & $60-80$ \\
\hline 40 & & Yozgat-24 & Karaveli-24 & Turkey & $39^{\circ} 38^{\prime} 5.43^{\prime \prime}$ & $35^{\circ} 13^{\prime} 11.08^{\prime \prime}$ & 1098 & $150-200$ \\
\hline 41 & & Yozgat-25 & Tuzlacik-25 & Turkey & $39^{\circ} 35^{\prime} 23.10^{\prime \prime}$ & $35^{\circ} 17^{\prime} 20.46^{\prime \prime}$ & 1142 & $150-200$ \\
\hline 55 & & Yozgat-39 & Bahadin-39 & Turkey & $39^{\circ} 40^{\prime} 40.69^{\prime \prime}$ & $35^{\circ} 18^{\prime} 14.05^{\prime \prime}$ & 1097 & $40-50$ \\
\hline 56 & & Yozgat-40 & Bahadin-40 & Turkey & $39^{\circ} 40^{\prime} 38.77^{\prime \prime}$ & $35^{\circ} 18^{\prime} 12.72^{\prime \prime}$ & 1104 & $60-80$ \\
\hline 57 & & Yozgat-41 & Bahadin-41 & Turkey & $39^{\circ} 41^{\prime} 8.46^{\prime \prime}$ & $35^{\circ} 18^{\prime} 5.23^{\prime \prime}$ & 1087 & $80-100$ \\
\hline 58 & Sorgun & Yozgat-42 & Bahadin-42 & Turkey & $39^{\circ} 40^{\prime} 34.83^{\prime \prime}$ & $35^{\circ} 18^{\prime} 2.02^{\prime \prime}$ & 1135 & $50-60$ \\
\hline 59 & Yozgat & Yozgat-43 & Bahadin-43 & Turkey & $39^{\circ} 40^{\prime} 28.61^{\prime \prime}$ & $35^{\circ} 18^{\prime} 3.36^{\prime \prime}$ & 1131 & $60-80$ \\
\hline 60 & Turkey & Yozgat-44 & Bahadin-44 & Turkey & $39^{\circ} 40^{\prime} 29.52^{\prime \prime}$ & $35^{\circ} 18^{\prime} 1.42^{\prime \prime}$ & 1132 & $50-60$ \\
\hline 61 & & Yozgat-45 & Karabali-45 & Turkey & $39^{\circ} 52^{\prime} 9.67^{\prime \prime}$ & $35^{\circ} 12^{\prime} 30.51^{\prime \prime}$ & 1220 & $80-100$ \\
\hline 62 & & Yozgat-46 & Karabali-46 & Turkey & $39^{\circ} 52^{\prime} 9.36^{\prime \prime}$ & $35^{\circ} 12^{\prime} 31.34^{\prime \prime}$ & 1218 & $70-80$ \\
\hline 63 & & Yozgat-47 & Karabali-47 & Turkey & $39^{\circ} 52^{\prime} 9.16^{\prime \prime}$ & $35^{\circ} 12^{\prime} 43.50^{\prime \prime}$ & 1219 & $60-80$ \\
\hline 64 & & Yozgat-48 & Karabali-48 & Turkey & $39^{\circ} 52^{\prime} 6.63^{\prime \prime}$ & $35^{\circ} 12^{\prime} 47.33^{\prime \prime}$ & 1219 & $60-80$ \\
\hline 65 & & Yozgat-49 & Karabali-49 & Turkey & $39^{\circ} 52^{\prime} 13.67^{\prime \prime}$ & $35^{\circ} 12^{\prime} 55.47^{\prime \prime}$ & 1229 & $50-60$ \\
\hline 66 & & Yozgat-50 & Karabali-50 & Turkey & $39^{\circ} 52^{\prime} 8.50^{\prime \prime}$ & $35^{\circ} 12^{\prime} 42.51^{\prime \prime}$ & 1221 & $70-80$ \\
\hline 67 & & Yozgat-51 & Karabali-51 & Turkey & $39^{\circ} 52^{\prime} 12.95^{\prime \prime}$ & $35^{\circ} 12^{\prime} 52.46^{\prime \prime}$ & 1218 & 100-120 \\
\hline 31 & & Yozgat-14 & Erkeklikoyu-14 & Turkey & $39^{\circ} 37^{\prime} 21.06^{\prime \prime}$ & $34^{\circ} 47^{\prime} 29.47^{\prime \prime}$ & 1177 & $60-80$ \\
\hline 32 & & Yozgat-15 & Basinayayla-15 & Turkey & $39^{\circ} 41^{\prime} 56.84^{\prime \prime}$ & $34^{\circ} 47^{\prime} 9.05^{\prime \prime}$ & 1280 & $50-60$ \\
\hline 33 & & Yozgat-16 & Basinayayla-16 & Turkey & $39^{\circ} 41^{\prime} 55.45^{\prime \prime}$ & $34^{\circ} 46^{\prime} 53.90^{\prime \prime}$ & 1296 & $50-60$ \\
\hline 34 & & Yozgat-17 & Basinayayla-17 & Turkey & $39^{\circ} 42^{\prime} 1.50^{\prime \prime}$ & $34^{\circ} 46^{\prime} 52.59^{\prime \prime}$ & 1286 & $50-60$ \\
\hline 35 & & Yozgat-18 & Basinayayla-18 & Turkey & $39^{\circ} 42^{\prime} 7.78^{\prime \prime}$ & $34^{\circ} 46^{\prime} 51.57^{\prime \prime}$ & 1280 & $50-60$ \\
\hline 36 & & Yozgat-19 & Basinayayla-19 & Turkey & $39^{\circ} 42^{\prime} 5.53^{\prime \prime}$ & $34^{\circ} 47^{\prime} 1.00^{\prime \prime}$ & 1280 & $50-60$ \\
\hline 37 & Yozgat & Yozgat-20 & Basinayayla-20 & Turkey & $39^{\circ} 42^{\prime} 1.66^{\prime \prime}$ & $34^{\circ} 47^{\prime} 10.10^{\prime \prime}$ & 1282 & $50-60$ \\
\hline 68 & Turkey & Yozgat-52 & Catak-52 & Turkey & $39^{\circ} 50^{\prime} 21.28^{\prime \prime}$ & $34^{\circ} 47^{\prime} 48.74^{\prime \prime}$ & 1470 & $80-100$ \\
\hline 69 & & Yozgat-53 & SeyhOsman-53 & Turkey & $39^{\circ} 50^{\prime} 26.08^{\prime \prime}$ & $34^{\circ} 47^{\prime} 55.05^{\prime \prime}$ & 1485 & 150-200 \\
\hline 70 & & Yozgat-54 & Bascavus-54 & Turkey & $39^{\circ} 49^{\prime} 23.90^{\prime \prime}$ & $34^{\circ} 48^{\prime} 8.40^{\prime \prime}$ & 1319 & $150-200$ \\
\hline 71 & & Yozgat-55 & Eskipazar-55 & Turkey & $39^{\circ} 49^{\prime} 51.95^{\prime \prime}$ & $34^{\circ} 47^{\prime} 59.87^{\prime \prime}$ & 1304 & $150-200$ \\
\hline 72 & & Yozgat-56 & Eskipazar-56 & Turkey & $39^{\circ} 48^{\prime} 52.68^{\prime \prime}$ & $34^{\circ} 48^{\prime} 5.06^{\prime \prime}$ & 1306 & 80-100 \\
\hline
\end{tabular}


Table 1. Cont.

\begin{tabular}{|c|c|c|c|c|c|c|c|c|}
\hline No & Location & $\begin{array}{l}\text { Sample } \\
\text { Code }\end{array}$ & Accessions & $\begin{array}{c}\text { Country of } \\
\text { Origin }\end{array}$ & Latitude & Longitude & $\begin{array}{l}\text { Altitude } \\
\text { (m) }\end{array}$ & $\begin{array}{c}\text { Tree Age } \\
\text { (year) }\end{array}$ \\
\hline 73 & & Yozgat-57 & Tekke-57 & Turkey & $39^{\circ} 48^{\prime} 57.74^{\prime \prime}$ & $34^{\circ} 48^{\prime} 15.28^{\prime \prime}$ & 1298 & $150-200$ \\
\hline 74 & & Yozgat-59 & Tayyipkoyu-59 & Turkey & $39^{\circ} 47^{\prime} 18.66^{\prime \prime}$ & $34^{\circ} 44^{\prime} 34.84^{\prime \prime}$ & 1216 & 80-100 \\
\hline 75 & & Yozgat- 60 & Tayyipkoyu-60 & Turkey & $39^{\circ} 50^{\prime} 14.83^{\prime \prime}$ & $34^{\circ} 43^{\prime} 36.64^{\prime \prime}$ & 1206 & $40-50$ \\
\hline 76 & & Yozgat-61 & Tayyipkoyu-61 & Turkey & $39^{\circ} 50^{\prime} 14.83^{\prime \prime}$ & $34^{\circ} 43^{\prime} 36.64^{\prime \prime}$ & 1209 & 80-100 \\
\hline 77 & & Yozgat-62 & Tayyipkoyu-62 & Turkey & $39^{\circ} 50^{\prime} 15.99^{\prime \prime}$ & $34^{\circ} 43^{\prime} 37.09^{\prime \prime}$ & 1206 & $70-80$ \\
\hline 78 & & Yozgat-63 & Tayyipkoyu-63 & Turkey & $39^{\circ} 50^{\prime} 17.12^{\prime \prime}$ & $34^{\circ} 43^{\prime} 35.69^{\prime \prime}$ & 1208 & $70-80$ \\
\hline 79 & & Yozgat-64 & Tayyipkoyu-64 & Turkey & $39^{\circ} 50^{\prime} 17.58^{\prime \prime}$ & $34^{\circ} 43^{\prime} 34.90^{\prime \prime}$ & 1208 & $80-100$ \\
\hline 80 & & Yozgat-65 & Kalekoyu-65 & Turkey & $39^{\circ} 52^{\prime} 23.27^{\prime \prime}$ & $34^{\circ} 40^{\prime} 19.33^{\prime \prime}$ & 1204 & $40-50$ \\
\hline 81 & & Yozgat-66 & Kalekoyu-66 & Turkey & $39^{\circ} 52^{\prime} 19.51^{\prime \prime}$ & $34^{\circ} 40^{\prime} 7.92^{\prime \prime}$ & 1173 & $40-50$ \\
\hline 82 & & Yozgat-68 & Kalekoyu-68 & Turkey & $39^{\circ} 52^{\prime} 20.03^{\prime \prime}$ & $34^{\circ} 40^{\prime} 1.15^{\prime \prime}$ & 1166 & $100-120$ \\
\hline 83 & & Yozgat-69 & Kalekoyu-69 & Turkey & $39^{\circ} 52^{\prime} 19.82^{\prime \prime}$ & $34^{\circ} 39^{\prime} 55.93^{\prime \prime}$ & 1151 & $80-100$ \\
\hline 84 & & Yozgat-70 & Kalekoyu-70 & Turkey & $39^{\circ} 52^{\prime} 19.21^{\prime \prime}$ & $34^{\circ} 39^{\prime} 54.99^{\prime \prime}$ & 1141 & $100-120$ \\
\hline 85 & & Yozgat-71 & Kalekoyu-71 & Turkey & $39^{\circ} 52^{\prime} 18.87^{\prime \prime}$ & $34^{\circ} 39^{\prime} 47.53^{\prime \prime}$ & 1139 & $40-50$ \\
\hline 86 & & Yozgat-73 & Musabeyli-73 & Turkey & $39^{\circ} 52^{\prime} 7.29^{\prime \prime}$ & $34^{\circ} 39^{\prime} 18.04^{\prime \prime}$ & 1027 & $80-100$ \\
\hline 87 & & Yozgat-74 & Musabeyli-74 & Turkey & $39^{\circ} 51^{\prime} 56.90^{\prime \prime}$ & $34^{\circ} 39^{\prime} 18.46^{\prime \prime}$ & 1056 & $60-80$ \\
\hline 88 & & Yozgat-75 & Musabeyli-75 & Turkey & $39^{\circ} 51^{\prime} 56.80^{\prime \prime}$ & $34^{\circ} 39^{\prime} 18.46^{\prime \prime}$ & 1057 & $40-50$ \\
\hline 89 & & Yozgat-76 & Musabeyli-76 & Turkey & $39^{\circ} 51^{\prime} 49.47^{\prime \prime}$ & $34^{\circ} 39^{\prime} 7.82^{\prime \prime}$ & 1008 & $50-60$ \\
\hline 90 & & Yozgat-77 & Musabeyli-77 & Turkey & $39^{\circ} 51^{\prime} 49.21^{\prime \prime}$ & $34^{\circ} 39^{\prime} 2.96^{\prime \prime}$ & 1004 & $70-80$ \\
\hline 91 & & Yozgat-78 & Musabeyli-78 & Turkey & $39^{\circ} 51^{\prime} 20.03^{\prime \prime}$ & $34^{\circ} 39^{\prime} 2.26^{\prime \prime}$ & 1091 & $80-100$ \\
\hline
\end{tabular}

$\left(^{*}\right)$ Refers to commercial cultivar.

\subsection{DNA Extraction}

Total genomic DNA was isolated from fresh leaves by the CTAB method described by Doyle and Doyle [32] with some modifications [11]. The Qubit Fluorometer (ThermoFisher Scientific, Waltham, MA, USA) was used to quantify isolated DNA. Followed by diluting the extracted DNA to $10 \mathrm{ng} / \mu \mathrm{L}$ for SSR-PCR reactions, the samples stored at $-20{ }^{\circ} \mathrm{C}$.

\subsection{SSR-PCR Reactions}

Eight walnut cultivars were used for testing amplification success and degree of polymorphism of forty-eight previously published SSR primer pairs (Table 2). Finally, 45 SSR primer pairs were selected for the characterization of 91 walnut accessions.

Table 2. Summary of SSR primer pairs developed from Juglans species in walnut accessions.

\begin{tabular}{|c|c|c|c|c|c|c|c|}
\hline No & $\begin{array}{l}\text { Origin and } \\
\text { Reference }\end{array}$ & Acronyms & $\begin{array}{c}\text { No. of } \\
\text { Tested Loci }\end{array}$ & $\begin{array}{l}\text { No. of Non- } \\
\text { Amplified } \\
\text { Loci }\end{array}$ & Monomorphic & Polymorphic & $\begin{array}{c}\text { Polymorphic } \\
\text { Rate (\%) }\end{array}$ \\
\hline 1 & Chen et al. (2013) & JMP & 1 & - & - & 1 & 100 \\
\hline 2 & Zhang et al. (2010) & WEST & 1 & - & - & 1 & 100 \\
\hline 3 & Zhang et al. (2013) & BFUJR & 1 & - & - & 1 & 100 \\
\hline 4 & Topcu et al. (2015) & $\begin{array}{l}\text { CUJRA, CUJRB, } \\
\text { CUJRD }\end{array}$ & 15 & 2 & - & 13 & 86.66 \\
\hline 5 & Ikhsan et al. (2016) & JRHR & 15 & - & - & 15 & 100 \\
\hline \multirow[t]{2}{*}{6} & Eser et al. (2018) & JRHR & 15 & 1 & - & 14 & 93.33 \\
\hline & Total & & 48 & 3 & - & 45 & \\
\hline
\end{tabular}

All SSR-PCR reactions were done based on a three-primer strategy according to Scheulke [33] with minor modifications. A total volume of $12.5 \mu \mathrm{L}$ containing $10 \mathrm{ng}$ DNA,

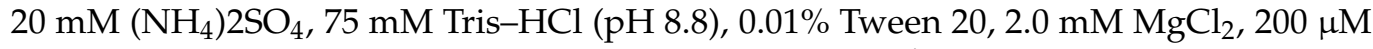
of each dNTP, $10 \mathrm{nM}$ M13 tailed forward primer at the $5^{\prime}$ end, $200 \mathrm{nM}$ reverse primer, 200 nM universal M13 tail primer (5'-TGTAAAACGACGGCCAGT-3') labeled with FAM, VIC, NED or PET dye, and 0.6 U of Taq DNA polymerase (New England BioLabs, Ipswich, MA) were used for each reaction. PCR amplifications were done in two consecutive steps. The first step included initial denaturation at $94{ }^{\circ} \mathrm{C}$ for $3 \mathrm{~min}$, followed by 28 cycles of 
$94{ }^{\circ} \mathrm{C}$ for $30 \mathrm{~s}, 58^{\circ} \mathrm{C}$ for $45 \mathrm{~s}$, and $72{ }^{\circ} \mathrm{C}$ for $60 \mathrm{~s}$. The second step involved 10 cycles of $94{ }^{\circ} \mathrm{C}$ for $30 \mathrm{~s}, 52{ }^{\circ} \mathrm{C}$ for $45 \mathrm{~s}$ and $72{ }^{\circ} \mathrm{C}$ for $60 \mathrm{~s}$, and a final extension at $72{ }^{\circ} \mathrm{C}$ for $5 \mathrm{~min}$. When the PCRs were completed, the reactions were subjected to denaturation for capillary electrophoresis in an ABI 3130xl genetic analyzer (Applied Biosystems Inc., Foster City, CA, USA (ABI)) using a 36-cm capillary array with POP7 as the matrix (ABI). Samples were denatured by mixing $0.5 \mu \mathrm{L}$ (in 6-FAM and VIC labeled primers) or $1.0 \mu \mathrm{L}$ (in NED and PET labeled primers) of the amplified product, $0.3 \mu \mathrm{L}$ of the size standard and $9.7 \mu \mathrm{L}$ of $\mathrm{Hi}-\mathrm{Di}$ formamide. The ABI data collection software 3.0 was used for resolving the fragments, and then SSR fragment analysis was done using the GeneMapper 4.0 (Applied Biosystems Inc. Bedford, MA, USA).

\subsection{Molecular Data Analysis}

\subsubsection{Genetic Diversity}

The effective number of alleles ( $\mathrm{Ne}$ ), expected heterozygosity $(\mathrm{He})$, the number of alleles per locus $(\mathrm{Na})$, and observed heterozygosity $(\mathrm{Ho})$ were calculated using the GenAlEx version 6.5 program [34]. PIC for the loci was calculated using PowerMarker software version 3.25 [35].

\subsubsection{Population Relationship}

The dendrogram, based on shared allele genetic distance was constructed using the UPGMA (Unweighted Pair Group Method with Arithmetic Average) method implemented in Molecular Evolutionary Genetics Analysis (MEGA) Program v. 10.2.2 [36]. Principal Coordinates (PCoA) based clustering was also done using the GenAlEx version 6.5 program. STRUCTURE 2.3.4, the model-based software, was used for population structure and identification of admixed individuals [37]. In this model, a number of populations (K) are considered to be available, which each of them is characterized by a set of allele frequencies at each locus. Individuals in the sample are given to populations (clusters), or jointly to more populations if their cultivars indicate that they are admixed. Ln P (D) values (logarithm probability for each $\mathrm{K}$ ) were applied to determine the Delta $\mathrm{K}$ indicating the probable population number. The term of Delta $\mathrm{K}$ is calculated by the change ratio of logarithm probability $(\Delta \mathrm{K}=2$ to $\Delta \mathrm{K}=10)$. In the diagram, the highest $\mathrm{K}$ of Delta $\mathrm{K}$ confers the information about the probable population number.

\section{Results}

\subsection{Polymorphism Levels of SSR Loci}

Of the screened 48 SSR primer pairs, three failed in the amplification, and the 45 remaining SSR markers generated polymorphic alleles for the eight walnut accessions tested, and they were consequently used for their genetic characterization (Table 2).

The genetic diversity analysis of walnut accessions included the average number of alleles (Na), number of effective alleles (Ne), observed heterozygosity (Ho), expected heterozygosity (He), and the polymorphism information content (PIC) (Table 3). Following the statistical analysis of the 45 polymorphic loci, in total, 390 alleles were detected for all studied accessions, and the number of alleles varied between 3 to 19 alleles per locus with a mean value of 9 . The highest number of allele was obtained from the CUJRA212 locus $(\mathrm{Na}=19)$. The number of effective alleles $(\mathrm{Ne})$ ranged from 1.89 (JRHR224485) to 7.92 (CUJRA212) with a mean of 4.06 . The observed heterozygosity (Ho) changed from 0.34 (JRHR225388) to 0.96 (JRHR227254) with a mean of 0.65. Observed heterozygosity (He) was the highest in the CUJRA212 loci. The average value of expected heterozygosity (He) was 0.73 and the highest value $(0.87)$ was seen in CUJRA212 locus. Polymorphism information content (PIC) of the loci ranged from 0.42 (JRHR222528 and JRHR224485) to 0.86 (CUJRA212) with an average of 0.68 (Table 3). 
Table 3. Sequence, allele range, number of alleles (Na), number of effective alleles (Ne), observed heterozygosity (Ho), expected heterozygosity (He), and polymorphism information content (PIC) values based on 45 SSR loci Juglans accessions.

\begin{tabular}{|c|c|c|c|c|c|c|c|c|c|}
\hline No & $\begin{array}{l}\text { Locus } \\
\text { Name }\end{array}$ & Sequence $\left(5^{\prime}-3^{\prime}\right)$ & Sequence $\left(5^{\prime}-3^{\prime}\right)$ & $\begin{array}{l}\text { Allele } \\
\text { Range }\end{array}$ & $\mathrm{Na}$ & $\mathrm{Ne}$ & Ho & He & PIC \\
\hline 1 & JRHR214458 & TTTCCTTAATAGCAGGTGTGTC & TATGTCCACCAAATGTATCCAC & $205-223$ & 9 & 4.03 & 0.69 & 0.75 & 0.70 \\
\hline 2 & CUJRD462 & TGCTCATTTTCATCCACTATC & АСТТССТСТССТТССТСТТТС & $252-269$ & 5 & 3.41 & 0.73 & 0.71 & 0.66 \\
\hline 3 & JRHR215674 & TCTAGAGGATCTCAAACGGACAT & ATTCTCCAACAGACGTAGCAATC & $166-176$ & 6 & 3.78 & 0.78 & 0.74 & 0.68 \\
\hline 4 & CUJRA202 & ACTCCAAAGTTTCTGGTATGG & AATAGTGGCAGTGAAGGATGTA & $180-198$ & 7 & 3.13 & 0.66 & 0.68 & 0.64 \\
\hline 5 & CUJRA218 & GTTTTTCTCTGTTGCCATCTC & GCTTCTTTGTTGAGGCTAATG & $241-269$ & 7 & 2.58 & 0.53 & 0.61 & 0.56 \\
\hline 6 & JRHR227254 & TGTAATTGGGAACTCACCTGAC & GGGGATGTAATGTGAATGGTCT & $187-220$ & 10 & 4.38 & 0.96 & 0.77 & 0.75 \\
\hline 7 & JRHR219728 & TTCTTGAATAACACTCTCTCСАTCC & AAGTGCAATGAGTTCTGTCTATGG & $267-283$ & 8 & 4.03 & 0.58 & 0.75 & 0.71 \\
\hline 8 & JRHR226941 & ATGCTATCGGCAACACCTAAA & CAGCAGAATTGCAAAATGACC & $201-227$ & 9 & 4.59 & 0.52 & 0.78 & 0.75 \\
\hline 9 & CUJRA212 & TGTCGCGGTGTTGTTCTAG & CGATTCAAGGAAGCAGCTC & $249-293$ & 19 & 7.92 & 0.42 & 0.87 & 0.86 \\
\hline 10 & JRHR223323 & AGTGTAACGGTGGTTCAATGC & ATCTGGTAAGATGGCCCTTTG & $202-220$ & 4 & 3.54 & 0.70 & 0.72 & 0.67 \\
\hline 11 & JRHR215721 & ACTACAATCACTGGGCTGAAAGA & TTGAGAACGGAGAACTCAGAAA & $207-221$ & 8 & 5.11 & 0.79 & 0.80 & 0.78 \\
\hline 12 & JRHR218769 & CTCСTCAATTCAGTCCСATCC & TTGCTCCCGTACTCTCAACTC & $182-228$ & 16 & 4.07 & 0.48 & 0.75 & 0.72 \\
\hline 13 & CUJRB321 & CGTAGTGGTCGTGTTTTGAC & GTTGGGTAAGTGTGAGAAGATG & $133-156$ & 13 & 5.38 & 0.67 & 0.81 & 0.78 \\
\hline 14 & JRHR231293 & TCTCCGAATTCTCTTCCTCAGT & TAAACTTGCTCTGGCAGGTGA & $184-212$ & 10 & 2.82 & 0.60 & 0.64 & 0.61 \\
\hline 15 & JRHR212270 & CTGACTGACCCTGCACATAAATA & ATTTCCTGCTACATGCATTTCC & $214-219$ & 5 & 2.81 & 0.60 & 0.64 & 0.56 \\
\hline 16 & JMP27 & TTCATAGCACATACCAGTTC & TCCGTAACATCAATCATTC & $308-334$ & 9 & 4.00 & 0.62 & 0.75 & 0.70 \\
\hline 17 & CUJRB453 & ACGAAAACATCATCTCAAACTC & СТССАССАСТСССТСТТС & $204-251$ & 12 & 3.93 & 0.64 & 0.75 & 0.70 \\
\hline 18 & JRHR230142 & GGGTTGTCGATGACAGAAAAT & GGATTGGGATGAGACACAAAA & $256-285$ & 5 & 3.14 & 0.38 & 0.68 & 0.58 \\
\hline 19 & CUJRA108 & ACCAACACAACCCACTGTAG & GGCATCTCTCTCTTCCATTT & $241-249$ & 6 & 2.07 & 0.55 & 0.52 & 0.48 \\
\hline 20 & JRHR227273 & ATGGTTATCAGCAGCTTACGG & CTGATTTTGATATCCCGTCCTT & $163-187$ & 6 & 4.18 & 0.77 & 0.76 & 0.72 \\
\hline 21 & JRHR222528 & CTGCTAGAGGCTAGGTTGGTTTG & GTCTGTGGCATAATCATCACCAT & $194-213$ & 6 & 1.92 & 0.42 & 0.48 & 0.42 \\
\hline 22 & CUJRA124 & CGTTGCCTGAACAAGTAAGAT & GAAGGAGGCTAACTCCСТATG & $165-186$ & 8 & 5.28 & 0.80 & 0.81 & 0.79 \\
\hline 23 & CUJRB441 & CAATCGGTCCATTTTTGTG & GTTTGCTTTCGTTATTTCTGG & $211-233$ & 10 & 6.13 & 0.75 & 0.84 & 0.81 \\
\hline 24 & JRHR223389 & GGTCGAAATTAAATCACATCCA & CGCAAGTCATGATACTGTCCA & $134-160$ & 9 & 4.13 & 0.65 & 0.76 & 0.71 \\
\hline 25 & JRHR229981 & CCGTCAATGGAGAGAGAGAGA & TTAAGTACGCGGGTAGGAAGG & $195-215$ & 6 & 2.26 & 0.45 & 0.56 & 0.50 \\
\hline 26 & JRHR223139 & AGATGAGCAAAACACGACACC & GCAACTCGATCCTCTTGCTTA & $225-243$ & 10 & 6.47 & 0.69 & 0.85 & 0.83 \\
\hline 27 & JRHR213115 & CGAGCAACAAGAGTACTACAGAAA & A GACTGTGCATGTGATGATGAGTA & $215-239$ & 9 & 4.26 & 0.69 & 0.77 & 0.73 \\
\hline 28 & JRHR209244 & ATGGTCTCGCGCGTTTATATT & ACAAGGACACGACCAATTATCAC & $150-186$ & 12 & 7.55 & 0.88 & 0.87 & 0.85 \\
\hline 29 & JRHR220176 & TGGTTACCTATCTTGATCATCTTCG & CGGATACCTCTGATTTTCGACTA & $222-248$ & 11 & 3.82 & 0.67 & 0.74 & 0.71 \\
\hline 30 & CUJRB220 & AGCATGTATAGGCCAATGATG & TCGTTCTATCTACAAGCACTCG & $147-196$ & 7 & 3.67 & 0.70 & 0.73 & 0.68 \\
\hline 31 & JRHR213218 & AATCTGTCTGTAACGTGGTTGGT & AACAGCCTTAGGAACTCGAAGAA & $177-217$ & 10 & 4.81 & 0.80 & 0.79 & 0.77 \\
\hline 32 & JRHR223620 & GGAAAAGCTGAGGAGACGGTA & AAACTGAGCTGCCCTTATTCC & $164-189$ & 9 & 4.09 & 0.76 & 0.76 & 0.72 \\
\hline 33 & CUJRB412 & ATCAAAATCСССАТСТСТTTC & GGGTGCTTTCCTCCTTGT & $144-159$ & 6 & 3.42 & 0.71 & 0.71 & 0.65 \\
\hline 34 & JRHR225388 & CATGTTAATCCATCCСТСТСAA & TCCGAATACCTCAGGAAGTTACA & $187-225$ & 13 & 5.50 & 0.34 & 0.82 & 0.78 \\
\hline 35 & BFUJR276 & TCGTGTGATTCTGGGTGTGT & GCCTCATTGGTTGAATTGCT & $163-201$ & 10 & 4.45 & 0.78 & 0.78 & 0.73 \\
\hline 36 & JRHR223518 & TACGTGAAGCTCCAATCATCC & CTTCCTATGACCGACCAGTGA & $180-198$ & 9 & 5.09 & 0.68 & 0.80 & 0.78 \\
\hline 37 & WEST1552 & GGGGAAGGGCAAGGAGTGCTCT & GCTATAAGCGAACCAATGCCCAG & $164-194$ & 5 & 3.30 & 0.66 & 0.70 & 0.64 \\
\hline 38 & JRHR229800 & CATGAGGGTCATTGGTTCAAG & TGCAAAGTCCATGTTTCTCTG & $184-195$ & 6 & 3.49 & 0.64 & 0.71 & 0.67 \\
\hline 39 & CUJRB111 & TAGGCATGTTCATCAAGGTC & GAGGAAGAAGTGCCAGTAGG & $191-330$ & 11 & 4.66 & 0.74 & 0.79 & 0.75 \\
\hline 40 & JRHR224485 & GGAGCTACTGGAGCGAGAAAC & CTTAAAGGTGTGGCTGTCGAG & $175-184$ & 3 & 1.89 & 0.52 & 0.47 & 0.42 \\
\hline 41 & JRHR227284 & CTGACCTGGGAGCAGAGAATA & CCGACAAGGAGTGCCTAGAAT & $194-208$ & 7 & 3.99 & 0.69 & 0.75 & 0.72 \\
\hline 42 & JRHR218727 & ATTCTTCAAATCCCACCATCC & TCCTTTAACGATAGATGAAGAGACC & $158-220$ & 13 & 1.96 & 0.53 & 0.79 & 0.47 \\
\hline 43 & CUJRD104 & GAGCCGATACTGCTGAACAG & ACGCGCATATCATAAACCTG & $217-226$ & 3 & 2.87 & 0.66 & 0.65 & 0.58 \\
\hline 44 & JRHR204109 & CAATTTGTGGCTGTATCACTCATC & ATGTACCACTAATCGCATTGCTC & $182-195$ & 10 & 4.91 & 0.63 & 0.80 & 0.77 \\
\hline \multirow[t]{5}{*}{45} & JRHR229005 & CCATTCCAGTCCATCTTCCTA & CCAAAGCAACAAGAATGGCTA & $215-275$ & 13 & 3.77 & 0.71 & 0.73 & 0.69 \\
\hline & Total & - & - & - & 390 & - & - & - & - \\
\hline & Min & - & - & - & 3 & 1.89 & 0.34 & 0.47 & 0.42 \\
\hline & Mean & - & - & - & 9 & 4.06 & 0.65 & 0.73 & 0.68 \\
\hline & $\operatorname{Max}$ & - & - & - & 19 & 7.92 & 0.96 & 0.87 & 0.86 \\
\hline
\end{tabular}

\subsection{Genetic Relationships among Walnut Accessions}

The generated dendrogram from the UPGMA analysis was shown in Figure 2a. Genetic dissimilarity coefficients ranged from 0.03 to 0.68 (Table S1). All walnut accessions were grouped into five significant clusters by the UPGMA clustering analysis. Cluster-I included USA (Serr, Chandler, Pedro, and Amigo), France (Fernor, Franquette, Fernette), and Unknown (Back) commercial walnut cultivars. Cluster-II included USA (Midland), and Turkey (Maras-18, Sutyemez-1, Yalova-1, Kaman-1, Kaplan-86, Bilecik-2, Bursa-95, Sebin-1, and Yozgat-12, 15, 17, 18, 19, 24, 54, 56, 66, 68, 69, 75, and, 78) accessions. Cluster-III, IV, and V included only Turkey accessions (Yozgat-1-Yozgat-11, Yozgat-13, 14, 16, 20, 21, 23, Yozgat-25-Yozgat-53, Yozgat-55, 57, Yozgat-59-Yozgat-65, Yozgat-70, 71, 73, 74, 76, 77) (Table S1; Figure 2a). 
a

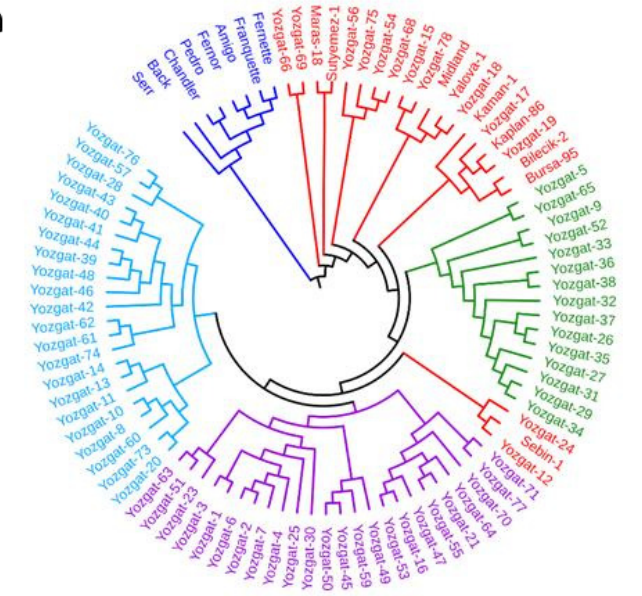

b

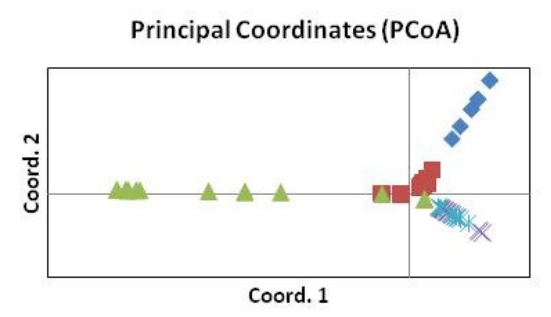

C

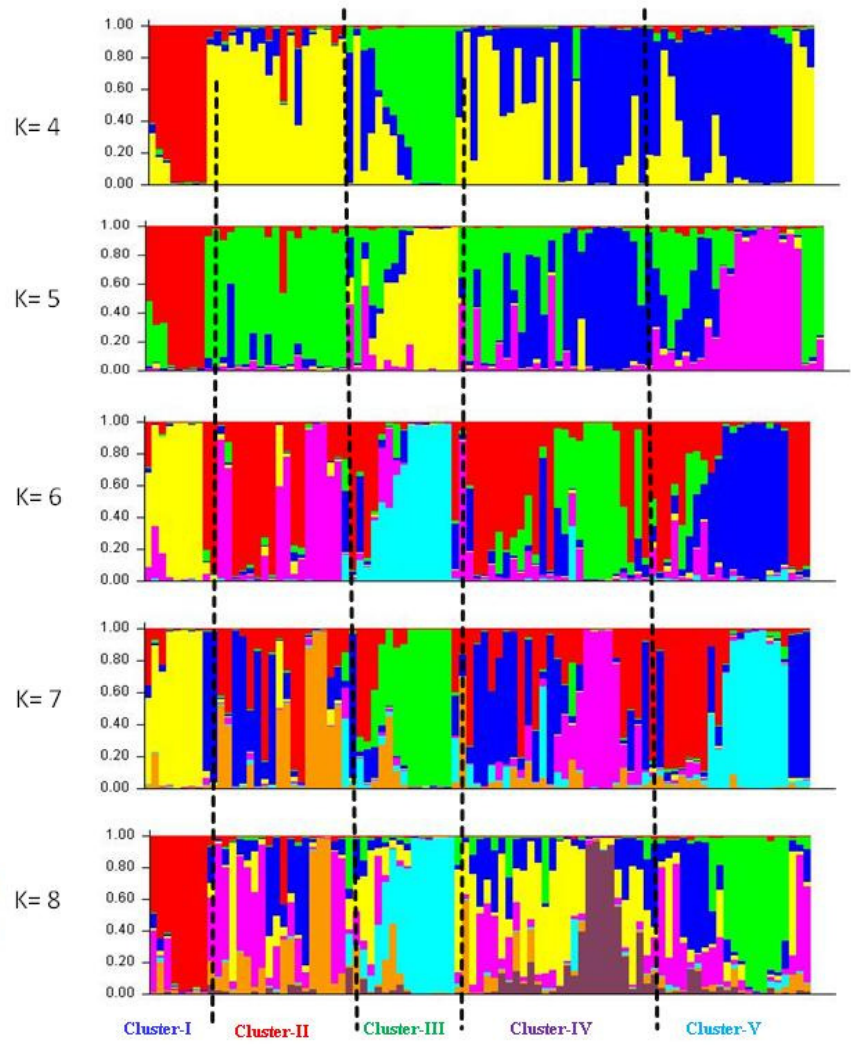

Figure 2. UPGMA, PCoA, and STRUCTURE $(\Delta K=5)$ analysis of 91 Juglans accessions. Each cluster group is color coded: Cluster-I included USA, French and Unknown cultivars (blue), Cluster-II included USA (Midland) and Turkish cultivars and accessions (red), and the remaining Turkish accessions were sorted into Cluster-III (green), Cluster-IV (purple), and Cluster-V (light blue). (a) Phylogenetic tree constructed using 45 SSRs. (b) Principal Component Analysis (PCoA) of the 91 samples. (c) STRUCTURE (K) from 4 to 8. Each vertical bar represents Juglans accessions cluster group.

The highest genetic dissimilarity coefficient of Cluster-I, Cluster-II, Cluster-III, ClusterVI, and Cluster-V was $0.07,0.03,0.23,0.2$, and 0.13 , respectively, which were achieved between Fernor and Amigo commercial walnut cultivars in Cluster-I, Kaplan-86 and Yozgat-19 in Cluster-II, Yozgat-35 and Yozgat-26 in Cluster-III, Yozgat-6 and Yozgat-2 in Cluster-VI, and Yozgat-44 and Yozgat-39 in Cluster-V (Table S1).

Principal Coordinate Analysis (PCoA) was done to envision the relationship between the cultivars in more detail. The variations expressed on axes 1, 2, and 3 were 4.60, 4.25 , and $3.01 \%$, respectively. The first principal component accounts for $11.86 \%$ of the total variance. Observation of the resultant matrix of PCoA (Figure 2b), showed that these walnut accessions were sorted into five main clusters. PCoA Cluster-I included eight commercial cultivars (USA, France, and Unknown), PCoA Cluster-II included nine commercial cultivars (USA and Turkey) and 13 accessions (Turkey), and PCoA Cluster-III, IV, and V included a totally 61 accessions (Turkey). Foreign commercial cultivars sorted into Cluster-I, and all Turkish commercial cultivars and one foreign cultivar (Midland) were sorted into Cluster-II. The remaining sixty-one accessions sorted into Cluster-III, IV, and V. The results of the PCoA showed that all accessions are separated from each other. Overall patterns of genetic differentiation denoted using the UPGMA (Figure 2a,b) and PCoA were in accordance with each other.

Structural genetic analysis was performed in 91 walnut accessions using 45 amplified loci by STRUCTURE and STRUCTURE HARVESTER programs. The highest value of Delta $\mathrm{K}(\Delta \mathrm{K})$ was obtained at $\Delta \mathrm{K}=5$ (Figure 3 ). $\Delta \mathrm{K}=5$ corresponded to the most possible number of populations in the study. Thus, all cultivars are categorized into five major clusters similar to the UPGMA and the PCoA based clustering results (Figure 2a,b). The dendrogram of the relationships of accessions was very similar to structural genetic analysis. 


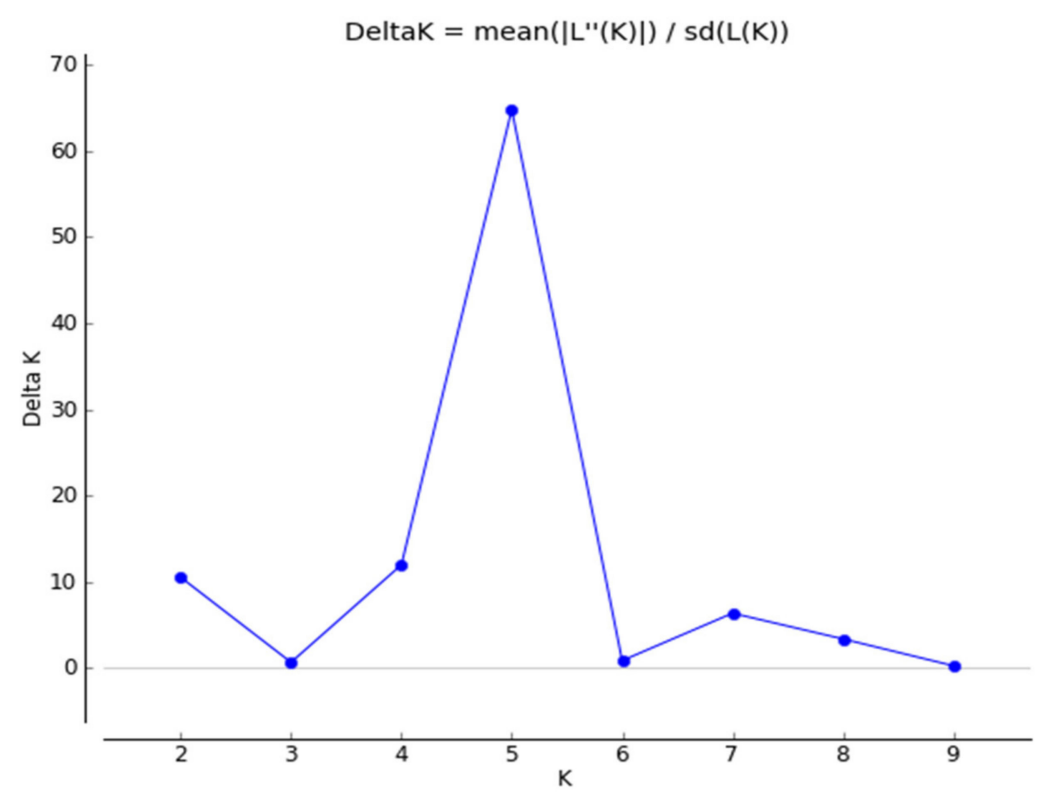

Figure 3. Value of $\Delta \mathrm{K}$ estimated for the structure analysis of ninety-one Juglans accessions $(\Delta \mathrm{K}=5)$.

\section{Discussion}

Molecular characteristic was used to elucidate the genetic diversity of 91 accessions.

\subsection{SSR Polymorphism}

In previous studies, Eser et al. [30], Dangl et al. [38], Karimi et al. [39], Pollegioni et al. [40], Ruiz-Garcia et al. [41], Kim et al. [42], Mahmoodi et al. [43], Pop et al. [44], Vahdati et al. [45], Bernard et al. [46], Khokhlov et al. [47], Balapanov et al. [48], and Orhan et al. [49] used SSRs to determine high genetic diversity and relationships of cultivated walnut accessions. We also present here high polymorphic SSR loci among walnuts (Tables 2 and 3).

Several studies revealed that SSR loci can be beneficial to evaluate molecular fingerprinting of Juglandaceae family species as Juglans regia. For example, in a study by Orhan et al. [49], a total of 135 polymorphic alleles with an average of 6.43 alleles per locus were obtained from 21 SSR primers among the 32 local divers walnut genotypes. Balapanov et al. [48] tested 11 SSR primers for 62 walnut genotypes and a total of 104 alleles were obtained in walnut tested genotypes with a mean value of 9.4. In another study, seven SSR loci were polymorphic in 15 diverse walnut cultivars from Ukraine. A total of 69 alleles were produced with an average of eight alleles per locus [47]. Bernard et al. [46] tested 13 SSR primers for 217 worldwide accessions and 116 alleles were detected with a mean value of 8.9. In an experiment by Vahdati et al. [45], 17 SSR primers were tested for six walnut populations and 147 alleles were detected with a mean value of 5.16. Pop et al. [44] tested seven polymorphic SSR primers for 20 walnut cultivars and reported 6.7 alleles per locus. Mahmoodi et al. [43] tested 16 walnut accessions and five cultivars using nine SSR markers, and they reported 34 alleles with a mean of 4.25. Kim et al. [42] tested eight Korean and 12 foreign walnut cultivars using 12 SSR primer pairs and they obtained an average of 9.6 alleles per locus. In a study, a total of 97 alleles were generated by 32 SSR loci with an average of five alleles per locus [41]. Pollegioni et al. [40] examined 29 Italian walnut genotypes using 12 SSR primers and they obtained a total of 62 alleles and an average of 6.2 alleles per locus. Karimi et al. [39] detected 63 alleles with a mean value of 5.73 from 11 SSR primers in the genetic structure of 105 walnut individuals. Moreover, Dangl et al. [38] tested 14 SSRs for 44 walnut genotypes and the number of alleles per locus ranged from three to eight with an average of 5.2. In the present study, 390 alleles with an average of nine alleles per locus were detected in the genetic characterization of ninety-one accessions. However, Kim et al. [42] and Balapanov et al. [48] reported the highest number of averages as 9.6 and 9.4, respectively. Although there are minor differences in polymorphic alleles 
in different studies due to the selection of accessions or cultivars, the results obtained in this study are in agreement with previous studies on genetic diversity in walnut accessions achieved by SSRs. Genetic and phylogenetic distance within the genotypes may also affect the polymorphism level detected by the SSR markers due to their out crossing nature.

Our results showed that the level of detected diversity was higher than many studies in the literature, with an average of expected and observed heterozygosities per locus. The reasons for this could be explained by specific characteristics of our populations because they obtained from seeds and showed great diversity due to open pollination.

In the current study, the average He and Ho were found as 0.73 and 0.65 , respectively, while Orhan et al. [49] reported them as 0.62 and 0.60, Balapanov et al. [48] found them as 0.75 and 0.67 , Khokhlov et al. [47] calculated them as 0.80 and 0.73, Bernard et al. [46] reported them as 0.56 and 0.47, Vahdati et al. [45] found 0.79 and 0.23, Pop et al. [44] calculated them as 0.72 and 0.65 , Mahmoodi et al. [43] reported them as 0.62 and 0.63 , Ruiz-Garcia et al. [41] found them as 0.57 and 0.51, Pollegioni et al. [40] reported them as 0.64 and 0.60 , and Karimi et al. [39] calculated them as 0.66 and 0.68. As a result, in this study, the average He and Ho were found higher compared to many studies in the literature. This might be due to using accessions that were wildly grown by seeds in a region that contains rich genetic resources that exhibited high heterozygosis. Although the genetic diversity of perennial species has resulted in fundamental changes in the mode of reproduction, cultivated walnuts are probably obtained from the selection of seedlings belong to different geographical regions of natural populations over years [50].

Polymorphism information content (PIC) refers to the value of a marker for detecting polymorphism within a population, depending on the number of detectable alleles and the distribution of their frequency [11]. The number of expressed alleles and frequency of alleles per locus which are equivalent to genetic diversity was determined by PIC of which is considered. In the current study, the PIC values were between 0.42 and 0.86 with an average of 0.68 , while Orhan et al. [49] reported 0.54 and 0.85 with an average of 0.68 , Bernard et al. [46] calculated 0.15 and 0.75 with an average of 0.52 , Vahdati et al. [45] found 0.56 and 0.82 with an average of 0.72 , Pop et al. [44] reported 0.51 and 0.83 with an average of 0.66 , Mahmoodi et al. [43] calculated 0.49 and 0.75 with an average of 0.64 , Pollegioni et al. [40] found 0.35 and 0.71 with an average of 0.57, and Karimi et al. [39] reported 0.49 and 0.85 with an average of 0.68 . As a result, in this study, the average PIC was found lower compared to the study conducted by Vahdati et al. [45]. The differences among studies may derive from using different SSR markers, or the locations of samples that may collect from much more diverse areas, even different continents [51]. However, higher genetic diversity values were obtained in this study compared to other studies in the literature. Pollegioni et al. [52] also reported a clear longitudinal trend of walnut genetic diversity in Eurasia, with loss of allelic richness and heterozygosity in Europe and reducing effective population size. However, the researchers reported a high degree of genetic diversity in walnuts in the Eurasia region including Turkey. These results supported the high genetic diversity and allelic richness exhibited in this study. Because, firstly, Turkey is located in the eastern Mediterranean basin survived after the Last Glacial Maximum, which sheltered rich genetic resources [53], secondly accessions collected from the Yozgat province have not been studied before and are unique wild accessions. However, the evolution of walnuts during decades also accounts for genetic diversity among accessions and cultivars. Although humans played a role in shaping the modern genetic structure, biogeographic events such as climate changes and socio-economic pressures should not be neglected in the evolution of walnut [53]. Productivity of adult walnut trees for at least 40 years, and surviving the nuts up to two years under simple storage conditions makes it easier to be transported short- and long-distance by humans causing the wide distribution of walnut worldwide [50].

On the other hand, the introduction of walnut to its new habitats may be dispersed easier by similarities in human language over large geographic areas. This may lead to the genetic homogenization of different populations known as the human role in walnut 
evolution by merging the plant biology and germplasm dispersal processes data with human cultural and linguistic diversity [54].

\subsection{Genetic Relationships among Walnut Accessions}

The results of SSR-based structural genetic analysis, the UPGMA, and the PCoA based clustering were similar to the genetic relationships of walnut accessions (Figure 2a-c). Seventeen commercial cultivars were collected from different origins (USA, French, Unknown, and Turkey). As reported by Orhan et al. [49], Khokhlov et al. [47], Pop et al. [44], Mahmoodi et al. [43], Kim et al. [42], Ruiz-Garcia et al. [41] some of these commercial cultivars were clustered in same groups which agreed with the reports of the current study (Figure 2). This result in this study provides that SSRs used in this study were enough to discriminate against the cultivars. The powerful dendrogram was obtained in this study that matches relationships reported among commercial walnut cultivars in previous studies [41-43,49]. The results are also imply that geographic barriers have shaped the distribution of walnut genetic resources.

PCoA results in the current study also proved that there is a high level of genetic diversity in walnut accessions studied in this paper. Previous studies also indicated efficacy of PCoA used in walnut and in different plant species [46,48,55-64].

\section{Conclusions}

In this study, walnut accessions analyzed by three different genetic analyses revealed that all ninety-one cultivars, which were fingerprinted using 45 SSR markers, were grouped at clusters with the same geographic origins. The assessment of the molecular diversity of walnut accessions is considerable for the optimal development of programs aiming the conservation of cultivated and wild genotypes in their ecosystems. SSR markers were distinctively capable of identifying all the studied walnut accessions in this study and proved that SSR markers are a potential tool for utilization in walnut breeding programs, genetic diversity, and germplasm characterization. These results could provide essential information for further understanding the genetic differentiation and utilization strategies for walnut germplasm. These markers as trustworthy tools can be applied for the evaluation of genetic diversity and relationships of walnut accessions in future molecular studies.

Supplementary Materials: The following are available online at https:/ /www.mdpi.com/article/10 $.3390 /$ su13126830/s1, Table S1: Walnut Table.

Author Contributions: M.G., S.K. and M.Z. were responsible for in silico SSRs and SSR data analysis. M.G., S.E., T.N. and G.B. coordinated and organized all research activities. M.Z., H.K. and M.A.G. performed DNA extractions and PCR reactions. All authors contributed to writing and editing the manuscript. All authors read and approved the published version of the manuscript.

Funding: The authors thank the Yozgat Bozok University Scientific Research Projects Unit (Project No. 6602c-ZF/19-255) for financial support.

Institutional Review Board Statement: Not applicable.

Informed Consent Statement: Not applicable.

Data Availability Statement: All-new research data were presented in this contribution.

Conflicts of Interest: The authors declare that they have no conflict of interest. 


\section{References}

1. Manning, W.E. The classification within the Juglandaceae. Ann. Mo. Bot. Gard. 1978, 65, 1058-1087. [CrossRef]

2. Woodworth, R.H. Meiosis of microsporogenesis in the Juglandaceae. Am. J. Bot. 1930, 17, 863-869. [CrossRef]

3. Simsek, M.; Gulsoy, E.; Beyhan, O.; Osmanoglu, A.; Turgut, Y. Determintion of some botanical, phenological, physical, and chemical characteristics of walnut (Juglans regia L.) genotypes grown in Turkey. Appl. Ecol. Environ. Res. 2017, 15, 1279-1291. [CrossRef]

4. Kafkas, E.; Attar, S.H.; Gundesli, M.A.; Ozcan, A.; Ergun, M. Phenolic and fatty acid profile, and protein content of different walnut cultivars and genotypes (Juglans regia L.) grown in the USA. Int. J. Fruit Sci. 2020, 20, S1711-S1720. [CrossRef]

5. Rahimipanah, M.; Hamedi, M.; Mirzapour, M. Antioxidant activity and phenolic contents of Persian walnut (Juglans regia L.) green husk extract. Afr. J. Food Sci. Technol. 2010, 1, 105-111.

6. Ros, E.; Mataix, J. Fatty acid composition of nuts-implications for cardiovascular health. Br. J. Nutr. 2006, 96, S29-S35. [CrossRef]

7. Faostat. FAO Web Page. Available online: http://www.fao.org/faostat (accessed on 7 September 2019).

8. Francesca, P.I.; Doru, P.; Raica, P.; Petricele, V.I.; Sisea, C.; Vas, E.; Botos, B.; Bodea, M.; Botu, M. Assessment of the genetic variability among some Juglans cultivars from the Romanian National Collection at SCDP Vâlcea using RAPD markers. Rom. Biotechnol. Lett. 2010, 15, 41-49.

9. Tie, L.; Feng, M.; Huang, C.; Peñuelas, J.; Sardans, J.; Bai, W.; Han, D.; Wu, T.; Li, W. Soil cover improves soil quality in a young walnut forest in the Sichuan Basin, China. Forests 2021, 12, 236. [CrossRef]

10. Orman, E.; Ates, D.; Ozkuru, E.; Hepaksoy, S.; Kafkas, S.; Tanyolac, M.B. Association mapping of several nut characters in walnut (Juglans regia L.). Turk. J. Agric. For. 2020, 44, 208-227. [CrossRef]

11. Guney, M.; Kafkas, S.; Keles, H.; Aras, S.; Ercisli, S. Characterization of hawthorn (Crataegus spp.) genotypes by SSR markers. Physiol. Mol. Biol. Plants 2018, 24, 1221-1230. [CrossRef] [PubMed]

12. Kafkas, S. Advances in Breeding of Pistachio; Burleigh Dodds Science Publishing: Cambridge, UK, 2019 ; pp. 389-430.

13. Guney, M.; Kafkas, S.; Koc, A.; Aras, S.; Keles, H. Characterization of quince (Cydonia oblonga Mill.) accessions by simple sequence repeat markers. Turk. J. Agric. For. 2019, 43, 69-79. [CrossRef]

14. Guney, M.; Kafkas, S.; Zarifikhosroshahi, M.; Gundesli, M.A.; Ercisli, S.; Holubec, V. Genetic diversity and relationships of terebinth (Pistacia terebinthus L.) genotypes growing wild in Turkey. Agronomy 2021, 11, 671. [CrossRef]

15. Hormaza, J. Molecular characterization and similarity relationships among apricot (Prunus armeniaca L.) genotypes using simple sequence repeats. Theor. Appl. Genet. 2002, 104, 321-328. [CrossRef] [PubMed]

16. Potts, S.M.; Han, Y.; Khan, M.A.; Kushad, M.M.; Rayburn, A.L.; Korban, S.S. Genetic diversity and characterization of a core collection of malus germplasm using Simple Sequence Repeats (SSRs). Plant Mol. Biol. Rep. 2012, 30, 827-837. [CrossRef]

17. Fan, L.; Zhang, M.-Y.; Liu, Q.-Z.; Li, L.T.; Song, Y.; Wang, L.-F.; Zhang, S.-L.; Wu, J. Transferability of newly developed pear SSR markers to other rosaceae species. Plant Mol. Biol. Rep. 2013, 31, 1271-1282. [CrossRef] [PubMed]

18. Zaloglu, S.; Kafkas, S.; Dogan, Y.; Guney, M. Development and characterization of SSR markers from pistachio (Pistacia vera L.) and their transferability to eight Pistacia species. Sci. Hortic. 2015, 189, 94-103. [CrossRef]

19. Woeste, K.; Burns, R.; Rhodes, O.; Michler, C. Thirty polymorphic nuclear microsatellite loci from black walnut. J. Hered. 2002, 93, 58-60. [CrossRef] [PubMed]

20. Victory, E.R.; Glaubitz, J.C.; Rhodes, O.E., Jr.; Woeste, K.E. Genetic homogeneity in Juglans nigra (Juglandaceae) at nuclear microsatellites. Am. J. Bot. 2006, 93, 118-126. [CrossRef]

21. Hoban, S.; Anderson, R.; McCleary, T.; Schlarbaum, S.; Romero-Severson, J. Thirteen nuclear microsatellite loci for butternut (Juglans cinerea L.). Mol. Ecol. Resour. 2008, 8, 643-646. [CrossRef] [PubMed]

22. Zhang, R.; Zhu, A.; Wang, X.; Yu, J.; Zhang, H.; Gao, J.; Cheng, Y.; Deng, X. Development of Juglans regia SSR markers by data mining of the EST database. Plant Mol. Biol. Rep. 2010, 28, 646-653. [CrossRef]

23. Feng, Y.; Zhang, Z.; Zhang, S.; Luo, S. Development of walnut EST-SSR markers and primer design. Agric. Sci. Technol. Hunan 2011, 12, 1810-1813.

24. Zhang, Z.Y.; Han, J.W.; Jin, Q.; Wang, Y.; Pang, X.M.; Li, Y.Y. Development and characterization of new microsatellites for walnut (Juglans regia). Genet. Mol. Res. 2013, 12, 4723-4734. [CrossRef]

25. Najafi, F.; Mardi, M.; Fakheri, B.; Pirseyedi, S.M.; Mehdinejad, N.; Farsi, M. Isolation and characterization of novel microsatellite markers in walnut (Juglans regia L.). Am. J. Plant Sci. 2014, 5, 409-415. [CrossRef]

26. Topcu, H.; Coban, N.; Woeste, K.; Sutyemez, M.; Kafkas, S. Developing new microsatellite markers in walnut (Juglans regia L.) from Juglans nigra genomic GA enriched library. Ekin J. Crop. Breed. Genetic. 2015, 1, 93-99.

27. Topcu, H.; Ikhsan, A.S.; Sutyemez, M.; Çoban, N.; Guney, M.; Kafkas, S. Development of 185 polymorphic simple sequence repeat (SSR) markers from walnut (Juglans regia L.). Sci. Hortic. 2015, 194, 160-167. [CrossRef]

28. Ikhsan, A.S.; Topcu, H.; Sutyemez, M.; Kafkas, S. Novel 307 polymorphic SSR markers from BAC-end sequences in walnut (Juglans regia L.): Effects of motif types and repeat lengths on polymorphism and genetic diversity. Sci. Hortic. 2016, $213,1-4$. [CrossRef]

29. Dang, M.; Zhang, T.; Hu, Y.; Zhou, H.; Woeste, K.E.; Zhao, P. De novo assembly and characterization of bud.; leaf and flowers transcriptome from Juglans regia L. for the identification and characterization of new EST-SSRs. Forests 2016, 7, 247. [CrossRef]

30. Eser, E.; Topcu, H.; Kefayati, S.; Sutyemez, M.; Islam, M.R.; Kafkas, S. Highly polymorphic novel simple sequence repeat markers from Class I repeats in walnut (Juglans regia L.). Turk. J. Agric. For. 2019, 43, 174-183. [CrossRef] 
31. Groover, A.T. Will genomics guide a greener forest biotech? Trends Plant. Sci. 2007, 12, 234-238. [CrossRef] [PubMed]

32. Doyle, J.J.; Doyle, J.L. A Rapid isolation procedure for small quantities of fresh leaf tissue. Phytochem. Bull. 1987, 19, 11-15.

33. Schuelke, M. An economic method for the fluorescent labeling of PCR fragments. Nat. Biotechnol. 2000, 18, 233-234. [CrossRef] [PubMed]

34. Peakall, R.; Smouse, P.E. Genalex 6: Genetic analysis in Excel. Population genetic software for teaching and research. Mol. Ecol. Notes 2006, 6, 288-295. [CrossRef]

35. Liu, K.; Muse, S.V. PowerMarker: Integrated Analysis Environment for Genetic Marker Data. Bioinformatics 2005, 21, 2128-2129. [CrossRef] [PubMed]

36. Kumar, S.; Stecher, G.; Li, M.; Knyaz, C.; Tamura, K. MEGA X: Molecular Evolutionary Genetics Analysis across Computing Platforms. Mol. Biol. Evol. 2018, 35, 1547-1549. [CrossRef]

37. Pritchard, J.K.; Stephens, M.; Donnelly, P. Inference of population structure using multilocus genotype data. Genetics 2000, 155, 945-959. [CrossRef] [PubMed]

38. Dangl, G.S.; Woeste, K.; Aradhya, M.K.; Koehmstedt, A.; Simon, C.; Potter, D.; Leslie, C.A.; McGranahan, G. Characterization of 14 microsatellite markers for genetic analysis and cultivar identification of walnut. J. Am. Soc. Hortic. Sci. 2005, 130, 348-354. [CrossRef]

39. Karimi, R.; Ershadi, A.; Vahdati, K.; Woeste, K. Molecular characterization of Persian walnut populations in Iran with microsatellite markers. HortScience 2010, 45, 1403-1406. [CrossRef]

40. Pollegioni, P.; Woeste, K.; Olimpieri, I.; Marandola, D.; Cannata, F.; Malvolti, M.E. Long-term human impacts on genetic structure of Italian walnut inferred by SSR markers. Tree Genet. Genomes 2011, 7, 707-723. [CrossRef]

41. Ruiz-Garcia, L.; López-Ortega, G.; Denia, A.; Tomas, D.F. Identification of a walnut (Juglans regia L.) germplasm collection and evaluation of their genetic variability by microsatellite markers. Span. J. Agric. Res. 2011, 9, 179-192. [CrossRef]

42. Kim, H.B.; Jeon, J.H.; Han, A.R.; Lee, Y.; Jun, S.S.; Kim, T.H.; Park, P.B. Genetic evaluation of domestic walnut cultivars trading on Korean tree markets using microsatellite markers. Afr. J. Biotechnol. 2012, 11, 7366-7374. [CrossRef]

43. Mahmoodi, R.; Rahmani, F.; Rezaee, R. Genetic diversity among Juglans regia L. genotypes assessed by morphological traits and microsatellite markers. Span. J. Agric. Res. 2013, 11, 431-437. [CrossRef]

44. Pop, I.F.; Vicol, A.C.; Botu, M.; Raica, P.A.; Vahdati, K.; Pamfil, D. Relationships of walnut cultivars in a germplasm collection: Comparative analysis of phenotypic and molecular data. Sci. Hortic. 2013, 153, 124-135. [CrossRef]

45. Vahdati, K.; Pourtaklu, S.M.; Karimi, R.; Barzehkar, R.; Amiri, R.; Mozaffari, M.; Woeste, K. Genetic diversity and gene flow of some Persian walnut populations in southeast of Iran revealed by SSR markers. Plant Syst. Evol. 2015, 301, 691-699. [CrossRef]

46. Bernard, A.; Barreneche, T.; Lheureux, F.; Dirlewanger, E. Analysis of genetic diversity and structure in a worldwide walnut (Juglans regia L.) germplasm using SSR markers. PLoS ONE 2018, 13, e0208021. [CrossRef]

47. Khokhlov, S.; Panyushkina, E.; Balapanov, I.; Suprun, I.; Tokmakov, S. Identification of walnut cultivars from Nikita Botanical Gardens using SSR-markers. Acta Hortic. 2018, 1208, 47-52. [CrossRef]

48. Balapanov, I.; Suprun, I.; Stepanov, I.; Tokmakov, S.; Lugovskoy, A. Comparative analysis Crimean.; Moldavian and Kuban Persian walnut collections genetic variability by SSR-markers. Sci. Hortic. 2019, 253, 322-326. [CrossRef]

49. Orhan, E.; Eyduran, S.P.; Poljuha, D.; Akin, M.; Weber, T.; Ercisli, S. Genetic diversity detection of seed-propagated walnut (Juglans regia L.) germplasm from Eastern Anatolia using SSR markers. Folia Hortic. 2020, 32, 37-46. [CrossRef]

50. Pollegioni, P.; Woeste, K.E.; Chiocchini, F.; Del Lungo, S.; Olimpieri, I.; Tortolano, V.; Clark, J.; Hemery, G.E.; Mapelli, S.; Malvolti, M.E. Ancient humans influenced the current spatial genetic structure of common walnut populations in Asia. PLoS ONE 2015, 10, e0135980. [CrossRef]

51. Budak, H.; Shearman, R.C.; Parmaksiz, I.; Dweikat, I. Comparative analysis of seeded and vegetative biotype buffalograsses based on phylogenetic relationship using ISSRs.; SSRs.; RAPDs.; and SRAPs. Theor. Appl. Genet. 2004, 109, 280-288. [CrossRef]

52. Pollegioni, P.; Woeste, K.E.; Chiocchini, F.; Del Lungo, S.; Ciolfi, M.; Olimpieri, I.; Tortolano, V.; Clark, J.; Hemery, G.E.; Mapelli, S.; et al. Rethinking the history of common walnut (Juglans regia L.) in Europe: Its origins and human interactions. PLoS ONE 2017, 12, e0172541. [CrossRef]

53. Aradhya, M.; Velasco, D.; Ibrahimov, Z.; Toktoraliev, B.; Maghradze, D.; Musayev, M.; Bobokashvili, Z.; Preece, J.E. Genetic and ecological insights into glacial refugia of walnut (Juglans regia L.). PLoS ONE 2017, 12, e0185974. [CrossRef]

54. Pollegioni, P.; Lungo, S.D.; Müller, R.; Woeste, K.E.; Chiocchini, F.; Clark, J.; Hemery, G.E.; Mapelli, S.; Villani, F.; Malvolti, M.E.; et al. Biocultural diversity of common walnut (Juglans regia L.) and sweet chestnut (Castanea sativa Mill.) across Eurasia. Ecol. Evol. 2020, 10, 11192-11216. [CrossRef] [PubMed]

55. Ercisli, S.; Esitken, A.; Cangi, R.; Sahin, F. Adventitious root formation of kiwifruit in relation to sampling date, IBA and Agrobacterium rubi inoculation. Plant Growth Regul. 2003, 41, 133-137. [CrossRef]

56. Zia-Ul-Haq, M.; Ahmad, S.; Qayum, M.; Ercisli, S. Compositional studies and antioxidant potential of Albizia lebbeck (L.) Benth. Pods and seeds. Turk. J. Biol. 2013, 37, 25-32.

57. Dogan, H.; Ercisli, S.; Temim, E.; Hadziabulic, A.; Tosun, M.; Yilmaz, S.O.; Zia-Ul-Haq, M. Diversity of chemical content and biological activity in flower buds of a wide number of wild grown caper (Capparis ovate Desf.) genotypes from Turkey. C. R. Acad. Bulg. Sci. 2014, 67, 1593-1600.

58. Gundogdu, M.; Ozrenk, K.; Ercisli, S.; Kan, T.; Kodad, O.; Hegedus, A. Organic acids, sugars, vitamin C content and some pomological characteristics of eleven hawthorn species (Crataegus spp.) from Turkey. Biol. Res. 2014, 47, 1-5. [CrossRef] 
59. Ergun, Z.; Zarifikhosroshahi, M. A comparative analysis of oil content and fatty acid in different varieties of Arachis hypogaea L. from Turkey. Int. J. Agric. For. Life Sci. 2020, 4, 42-47.

60. Ergun, Z.; Bozkurt, T. Determination of fatty acid composition and antioxidant activity of fig seed oil. Int. J. Agric. Nat. Sci. 2020, $13,101-107$.

61. Gecer, M.K.; Kan, T.; Gundogdu, M.; Ercisli, S.; Ilhan, G.; Sagbas, H.I. Physicochemical characteristics of wild and cultivated apricots (Prunus armeniaca L.) from Aras valley in Turkey. Genet. Resour. Crop Evol. 2020, 67, 935-945. [CrossRef]

62. Kupe, M. Some ampelographic and biochemical characteristics of local grape accessions from Turkey. Genet. Belgrade 2020, 52, 513-525. [CrossRef]

63. Ozkan, G.; Ercisli, S.; Sagbas, H.I.; Ilhan, G. Diversity on fruits of wild grown european cranberrybush from Coruh valley in Turkey. Erwerbs Obstbau 2020, 62, 275-279. [CrossRef]

64. Tabasi, M.; Sheidai, M.; Hassani, D.; Koohdar, F. DNA fingerprinting and genetic diversity analysis with SCoT markers of Persian walnut populations (Juglans regia L.) in Iran. Genet. Resour. Crop Evol. 2020, 67, 1437-1447. [CrossRef] 